\title{
CDH1 Testing: Can it Predict the Prophylactic or Therapeutic Nature of Total Gastrectomy in Hereditary Diffuse Gastric Cancer?
}

\author{
Dimosthenis Ziogas, $\mathrm{MD}^{1}$ and Dimitrios $\mathrm{H}$. Roukos, $\mathrm{MD}^{2}$ \\ ${ }^{1}$ Department of Surgery, Ioannina University Hospital, Ioannina, Greece; ${ }^{2}$ Department of Surgery, Personalized Cancer \\ Medicine, Biobank, Ioannina University, Ioannina, Greece
}

Gastric cancer has a poor prognosis. Primary prevention is a major goal to reduce incidence and mortality, but it is still an illusion. There is an exception: the rare hereditary diffuse gastric cancer (HDGC). This syndrome can be both accurately predicted and successfully prevented. How?

If we could identify high-risk individuals in the general population and subsequently tailor an effective preventive intervention, then we could protect them by saving their lives. This achievement is now realistic, although it concerns a small only fraction of cases. Genetic testing in individuals with a family history of diffuse gastric cancer allows for identification of family members with inherited mutations at $C D H 1$ gene. These mutation carriers have a very high risk of developing diffuse gastric cancer ${ }^{1}$ and women have an additional lobular breast cancer risk. ${ }^{2}$ This HDGC syndrome is characterized by early onset and requires primary prevention at a young age. ${ }^{1,2}$

Because the mortality risk in patients diagnosed at advanced tumor stage is very high in the west, and there is no standard screening method to identify accurately the disease in $\mathrm{CDH} 1$ mutation carriers at early stage, ${ }^{3}$ prophylactic total gastrectomy (PTG) has been recommended. ${ }^{4}$

However, the number of patients with disease-causing CDH1 mutations published who have undergone PTG is very small. Is PTG an experimental approach? Why is it that $\mathrm{CDH} 1$ mutation positive testing can be considered a

(C) Society of Surgical Oncology 2009

Published Online: 28 July 2009

D. H. Roukos, MD

e-mail: droukos@cc.uoi.gr surrogate predictor of the presence of occult signet-ringcells/diffuse gastric cancer? The decision for PTG at a young age is too hard given the aggressiveness and adverse effects of total gastrectomy on quality of life (QOL). However, if the predictive power is very high and indicative of microscopic cancer foci undetectable by any other current imaging or endoscopic screening technique, then the decision for therapeutic rather than prophylactic total gastrectomy is much more convincing. If the decision for surgery is made, then why is total chosen and not subtotal or partial gastrectomy? If a patient is diagnosed with a small diffuse gastric cancer in antrum and at the same time carries $C D H 1$ mutations is the current standard subtotal gastrectomy sufficient procedure especially for him or a total gastrectomy is required and why?

The E-cadherin protein encoded by the $C D H 1$ gene is important in the molecular connections between adjacent cells in the stomach, breast, and other areas of the body. Loss of E-cadherin function, associated with $\mathrm{CDH}$ mutations, is observed in diffuse-type gastric cancer and invasive lobular breast cancer. Penetrance estimates suggest that individuals with E-cadherin germline mutations face a lifetime risk of approximately $75 \%$ of developing diffuse gastric cancer and women have an additional $39 \%$ risk of developing lobular breast cancer. ${ }^{1,2}$

Adenocarcinomas of the stomach are distinguished into intestinal-type and diffuse-type cancer. Approximately 40 years after this classification introduced by Lauren, a recent genome-wide association (GWA) study confirmed that the intestinal and diffuse types are two distinctly different subtypes of gastric cancer. ${ }^{5}$ The intestinal type appears to be associated with Helicobacter pylori infection, which may lead to atrophic gastritis, intestinal 
metaplasia, dysplasia, and invasive adenocarcinoma, and is particularly common in some high-risk geographic regions, including Japan. By contrast, the diffuse-type develops through a different pathway. The genetic background is more important than for the intestinal type and appears unrelated to the presence of $\mathrm{H}$. pylori. ${ }^{3}$ The Study Group of Millennium Genome Project for Cancer identified through this new GWA study that genetic variants (SNPs) at PSCA gene are associated with a small increased relative risk $(<2)$ of sporadic diffuse gastric cancer. $^{5}$

\section{HERITABILITY AND ENVIRONMENT INTERACTIONS NETWORKS}

Cancer initiation and metastasis are extremely more complicated biological processes than we had previously imagined. Most recent extensive genetic studies and GWA studies have revealed that a tremendous number of diseasecausing variants at multiple genes contribute to carcinogenesis. ${ }^{6}$ However, each of these alterations confers a modest only relative risk $(\mathrm{RR}<2)$; thus, at present this information is unreliable for the clinic for common complex disease as well as for gastric cancer. ${ }^{7}$

The future clinical success of cancer treatment is uncertain if no realistic innovation appears. Despite enthusiasm with the next generation whole-genome scans for faster and cheaper completion of the genetic catalogue of individual cancer types, multiple hurdles should be overcome. For example, the functional role of genes, proteins and other small molecules, including miRNAs has to be elucidated. Particularly the understanding of their interactions networks as well as the gene-environment interaction is currently beyond researcher's and clinician's comfort. Currently, there is no standard model to understand the extremely complex causal networks feedbacks loops. In an effort to map the genotype-phenotype relationship, the most popular model used is the Bayesian network model, but it is associated with a series of limitations. ${ }^{8}$

Mathematical or computational (in silico) models to predict genetic or transcripts interactions (transcriptome), intracellular signalling pathways proteins interactions (interactome), and interactions between primary tumor cells and stromal cells (microenvironment) are under development. ${ }^{9}$ However, these predictions are beyond the current state-of-the-art. It has become clear that not only a wide spectrum of phenotypic cancer diversity exists, but also the underlying genetic basis varies widely even among patients with the same tumor type and similar conventional clinicopathologic features. There has been a clearly nonlinear relationship between DNA changes and cancer phenotype. ${ }^{8}$ This complexity to predict phenotypic cancer diversity by genotyping explains the current limitation in development of both rousts biomarkers and molecularly targeting agents toward innovative personalized cancer management. ${ }^{8,9}$

In sharp contrast to the current limitations of predicting causal networks for sporadic cancer (no family history), heritable mutations at only one specific gene $(\mathrm{CDH})$ can cause diffuse gastric cancer. Most $\mathrm{CDH} 1$ mutation carriers will develop diffuse gastric cancer. This relatively simple and nearly linear relationship between $\mathrm{CDHI}$ and phenotype (diffuse gastric cancer) allows genetic testing for identifying $\mathrm{CDHl}$ mutation carriers. Once a mutations carrier has been identified, which is the optimal medical intervention?

\section{TOTAL GASTRECTOMY IN CDHI MUTATION CARRIERS: PROPHYLACTIC OR THERAPEUTIC?}

Prophylactic total gastrectomy has been recommended for $\mathrm{CDH} 1$ mutation carriers; however, the published data are still limited. Plausible explanations for rare performance of PTG include: 1) physicians and public community are less aware on HDGC; 2) mutation carriers are rarely found in the general population and account for approximately $3-5 \%$ among patients diagnosed with gastric cancer. ${ }^{6}$ it is difficult to convince "healthy" young individuals to undergo total gastrectomy and live a long time "without stomach." Indeed, complete removal of the stomach maybe associated with postoperative morbidity and adverse effects in QOL. However, if $\mathrm{CDH} 1$ mutation testing could be a sole surrogate predictor of the presence of microscopic disease, then it might change the decision from prophylactic to therapeutic total gastrectomy.

To evaluate further all of these issues, Hebbard and colleagues reported the results of a retrospective study on PTG published in the July 2009 issue of the Annals of Surgical Oncology. ${ }^{10}$ Twenty-three patients from the Canadian province of Newfoundland and Labrador underwent PTG between February 2006 and November 2008. All of these individuals were confirmed to have a truncating mutation of the $\mathrm{CDHl}$ gene. The median age at the time of surgery was 45 (range 26-63) years. The patients came from three different kindreds, two of which shared the same 2398delC mutation of exon 15 of $\mathrm{CDH}$. The third family carried $1189 \mathrm{~A}>\mathrm{T}$ mutation in exon 9 of CDH1.

Preoperative endoscopy and mucosal biopsies revealed the disease in only 2 of 23 patients (9\%). Interestingly, final standardized pathological evaluation of TG specimens showed evidence of diffuse/signet-ring-cell carcinoma in 22 of $23(96 \%)$ patients. 
Despite its retrospective nature, the study by Hebbard and colleagues ${ }^{10}$ has important clinical implications. It provides a series of advantages. First, it is the largest series on PTG in HDGC prevention setting. Given the rarity of the syndrome, the number of $\mathrm{CDH} 1$ mutation carriers ( $n=23$ ) who consecutively underwent PTG within a relative short period (33 months) is reported for the first time. How can this large number of PTG in this study in the Canadian province of Newfoundland be explained? This region, according to recent estimates, has one of the highest HDGC rate due to both founder and independent mutations; approximately $40 \%$ of all gastric cancer cases are hereditary gastric cancer compared with less than $5 \%$ in North America. ${ }^{2,4}$

Second, it confirms that in the vast majority of cases, mutation carriers already had microscopic intramucosal disease, which explains the small $(9 \%)$ proportion of disease revealed by preoperative endoscopic screening. Table 1 summarizes the results of PTG for the prevention of HDGC. Although larger studies are required to confirm this very large rate of therapeutic gastrectomy among CDH1 mutation carriers, the data available (Table 1) strongly indicate the presence of intramucosal disease in these individuals. Therefore, we can consider most (>90) mutation carriers as patients and not healthy individuals who will ever develop diffuse gastric cancer.

Why total and not partial gastrectomy? First, multifocal microscopic disease is very common as demonstrated by pathohistological data (Table 1). Second, high-quality preclinical evidence suggests that the genetic basis of the HDGC syndrome is so strong that earlier or later cancer will arise in residual gastric epithelium. Therefore, subtotal gastrectomy is not recommended. This fact also should be considered for patients with a preoperative diagnosis of diffuse gastric cancer and at the same time a family history. If $\mathrm{CDH} 1$ testing is positive, total gastrectomy could be considered. It should be noted, however, that clinical data supporting this preclinical evidence are still scarce.

What is the optimal age for total gastrectomy? It is unknown. Although a mean age of onset of 37 years is reported in the literature, the wide age range for gastric cancer diagnosis is between 16 and 82 years, which makes it difficult to counsel on screening starting age and the more critical PTG age. Kaurah et al. recommend PTG during the early 1920s for men and individualization for women mutation carriers because of the dietary ramifications of gastrectomy on pregnancy. ${ }^{2}$ This is a careful suggestion to save lives and is based on both the therapeutic nature of total gastrectomy in $>90 \%$ of mutation carriers, and the low sensitivity and failure of current screening, including endoscopy, positron emission tomographic scanning, or chromoendoscopy-directed biopsies. Because long-term results from larger studies are still lacking, at present an individualized approach for timing of total gastrectomy considering multiple variables should be discussed with each $\mathrm{CDH} 1$ mutation carrier. For women carriers, beyond the $75 \%$ diffuse gastric cancer, the additional $40 \%$ lifetime risk of lobular breast cancer should be considered.

\section{FUTURE EXPECTATIONS}

During the years to come, total gastrectomy will remain the preventive intervention of choice for family members who test positive for heritable mutations at $\mathrm{CDH} 1$. Current

TABLE 1 Published data on prophylactic total gastrectomy among E-cadherin mutation carriers

\begin{tabular}{|c|c|c|c|c|}
\hline Study & Year & $\begin{array}{l}\text { Total gastrectomy } \\
\text { (no. of patients) }\end{array}$ & $\begin{array}{l}\text { Pathology (no. of patients } \\
\text { with signet-ring cancer cells) }\end{array}$ & Multifocality \\
\hline Hebbard et al. & 2009 & 23 & $22 / 23(96 \%)$ & N/A \\
\hline Lynch et al. & 2008 & 17 & 13/17 (76.5\%) & N/A \\
\hline Carneiro et al. & 2004 & 9 & $9 / 9$ & $5 / 9(55.5 \%)$ \\
\hline Barber et al. & 2008 & 8 & $8 / 8$ & $7 / 8(87.5 \%)$ \\
\hline Norton et al. & 2007 & 6 & $6 / 6$ & $6 / 6$ \\
\hline Lewis et al. & 2001 & 6 & $6 / 6$ & $5 / 6(83.3 \%)$ \\
\hline Charlton et al. & 2004 & 6 & $6 / 6$ & $6 / 6$ \\
\hline Huntsman et al. & 2001 & 5 & $5 / 5$ & \\
\hline Chun et al. & 2001 & 5 & $5 / 5$ & $5 / 5$ \\
\hline Medina-Franco et al. & 2007 & 4 & $4 / 4$ & $4 / 4$ \\
\hline Newman et al. & 2006 & 2 & $0 / 2$ & 0 \\
\hline Chung et al. & 2007 & 1 & $1 / 1$ & $0 / 1$ \\
\hline Francis et al. & 2007 & 1 & $1 / 1$ & $1 / 1$ \\
\hline Total & & 93 & $86 / 93(92.5 \%)$ & $42 / 52(80.8 \%)$ \\
\hline
\end{tabular}


efforts are focused in two directions: clinical practice and innovative research.

Interestingly, in the field of clinical practice, laparoscopic or robotic surgery already provides exciting data for improving QOL outcomes. Less postoperative pain, faster recovery, shorter hospital stay, brisk return to professional work, and substantially better aesthetic results are some of the advantages of closed vs. open surgery. During the last decade, rapid progression from laparoscopic-assisted distal gastrectomy with mini-laparotomy to totally laparoscopic distal gastrectomy without mini-laparotomy has occurred and moved from pioneering in laparoscopic gastrectomy countries, such as Korea and Japan, to the United States and Europe. For totally laparoscopic total gastrectomy (TLTG) required for $\mathrm{CDH} 1$ mutation carriers, the progress is less rapid. However, with an explosion in technology refinement and increasing surgeon's experience it is expected that TLTG will be performed with safety and effectivity in highly specialized institutions. Given that at this very early stage, extensive lymphadenectomy is not required, TLTG becomes easier. The rarity of the syndrome and the need for a multidisciplinary team, beyond high-volume surgeons, including geneticists, gastroenterologists, oncologists, psychologists, and nutritionists, makes it difficult to provide high-quality management for high-risk families besides a few Comprehensive Cancer Centers.

In the field of translational research, the endeavor is focused on predicting and distinguishing among CDHI mutation carriers the approximately $25 \%$ who will not develop diffuse gastric cancer from the $75 \%$ who will be diagnosed with invasive diffuse gastric. The most plausible explanation is that genetic and nongenetic factors promote or reverse the large effect of $\mathrm{CDH} 1$ mutated gene on tumorigenesis. New resequencing and GWA studies will probably identify the modifier genes. However, the challenge will remain about how to predict the complex interactions between these modifier genes, between these and mutated $\mathrm{CDH} 1$ gene, as well as between all of these genes and environmental factors, including $H$. pylori infection. $^{6}$

\section{CONCLUSIONS}

Genetic testing for identifying $C D H 1$ mutation carriers is a surrogate predictor of intramucosal presence of diffuse/ signet-ring cancer cells. This usually multifocal microscopic disease is undetectable by current screening technology. Although longer follow-up data from larger studies are required, total gastrectomy may save the lives of $\mathrm{CDH} 1$ mutation carriers. The therapeutic rather than prophylactic nature of surgery in these disease-causing mutation carriers is supported by the pathological evidence of cancer foci in most of these "healthy" individuals. Yet, the optimal timing of surgery is unknown. Because of early onset, total gastrectomy is recommended at a young age. Extensive discussion and counselling is needed on an individualized basis to decide on the timing of surgery. Because laparoscopic surgery is rapidly evolving, totally laparoscopic total gastrectomy with its substantial QOL advantages, more $\mathrm{CDH} 1$ mutation carriers can be convinced of the benefits of "prophylactic" surgery. Nextgeneration sequencing, modern functional studies, and systems biology provide promise for understanding complex interactions between $C D H 1$ mutated gene and lowrisk genetic and nongenetic modifiers. Integrating all clinical and genetics-personal genomics data into probabilistic predictive network models, we might be able to distinguish high-risk from lower-risk for developing diffuse gastric cancer among $C D H 1$ mutation carriers. Such a prediction may have important clinical implications.

\section{REFERENCES}

1. Guilford P, Hopkins J, Harraway J, et al. E-cadherin germline mutations in familial gastric cancer. Nature. 1998;392:402-5.

2. Kaurah P, MacMillan A, Boyd N, et al. Founder and recurrent $\mathrm{CDH} 1$ mutations in families with hereditary diffuse gastric cancer. JAMA.. 2007;297:2360-72.

3. Roukos DH. Genome-wide association studies and aggressive surgery toward individualized prevention, and improved local control and overall survival for gastric cancer. Ann Surg Oncol. 2009;16:795-8.

4. Lynch HT, Kaurah P, Wirtzfeld D, et al. Hereditary diffuse gastric cancer: diagnosis, genetic counseling, and prophylactic total gastrectomy. Cancer. 2008;112:2655-63.

5. Study Group of Millennium Genome Project for Cancer, Sakamoto H, Yoshimura K, Saeki N, et al. Genetic variation in PSCA is associated with susceptibility to diffuse-type gastric cancer. Nat Genet. 2008;40:730-40.

6. Roukos DH. Assessing both genetic variation (SNPs/CNVs) and gene-environment interactions may lead to personalized gastric cancer prevention. Expert Rev Mol Diagn. 2009;9:1-6.

7. Roukos DH. Genome-wide association studies: how predictable is a person's cancer risk? Expert Rev Anticancer Ther. 2009;9:389-92.

8. Roukos DH. Personalized cancer diagnostics and therapeutics. Expert Rev Mol Diagn. 2009;9:227-9.

9. Roukos DH. Mea culpa with cancer-targeted therapy: new thinking and new agents design for novel, causal networks-based, personalized biomedicine. Expert Rev Mol Diagn. 2009;9:21721.

10. Hebbard PC, Macmillan A, Huntsman D, et al. Prophylactic total gastrectomy (PTG) for hereditary diffuse gastric cancer (HDGC): the Newfoundland Experience with 23 patients. Ann Surg Oncol. 2009;16:1890-5. 